\title{
Ground State Phase Diagram of the Extended Hubbard Model with Pair-Hopping Interaction in the Limit of Very Narrow Bandwidth
}

\author{
K.J. KAPCIA ${ }^{a, *}$ AND W.R. CZART ${ }^{b}$ \\ ${ }^{a}$ Institute of Physics, Polish Academy of Sciences, al. Lotników 32/46, 02-668 Warszawa, Poland \\ ${ }^{b}$ Faculty of Physics, Adam Mickiewicz University, Umultowska 85, 61-614 Poznań, Poland
}

\begin{abstract}
The extended Hubbard model with the pair-hopping interaction, i.e. the Penson-Kolb-Hubbard model, is one of the conceptually simplest phenomenological models for studying correlations and for description of superconductivity in very narrow-band systems with short-range, almost unretarded pairing. We present ground state phase diagrams of the model derived within the broken-symmetry Hartree-Fock approximation in the narrow-bandwidth regime and compare these results with the exact ones in the atomic limit for the limit of high dimensions. The investigation of the diagrams of the model show that results obtained within both approaches are consistent, although for the case of the finite single-electron hopping phases with magnetic long-range order also occur.
\end{abstract}

DOI: 10.12693/APhysPolA.130.617

PACS/topics: 71.10.Fd, 71.10.Hf, 74.20.-z, 74.25.Dw

\section{Introduction}

Superconductivity (SC) with very short coherence length is still interesting and intriguing phenomenon due to its possible relevance to high-temperature superconductors (cuprates, doped bismuthates, iron-based systems, fullerenes) and also to several other exotic superconducting materials (for a review, see [1-4] and references therein). It can also give insight into behaviour of strongly bound fermion pairs on the optical lattices.

The Penson-Kolb-Hubbard Hamiltonian considered in this work has the following form (e.g. [5-12]):

$$
\begin{aligned}
\hat{H} & =\frac{t}{\sqrt{z}} \sum_{\langle i, j\rangle, \sigma}\left(\hat{c}_{i \sigma}^{+} \hat{c}_{j \sigma}+\hat{c}_{j \sigma}^{+} \hat{c}_{i \sigma}\right)+U \sum_{i} \hat{n}_{i \uparrow} \hat{n}_{i \downarrow} \\
& -\frac{J}{z} \sum_{\langle i, j\rangle}\left(\hat{\rho}_{i}^{+} \hat{\rho}_{j}^{-}+\hat{\rho}_{j}^{+} \hat{\rho}_{i}^{-}\right)-\mu \sum_{i} \hat{n}_{i},
\end{aligned}
$$

where $\hat{n}_{i}=\sum_{\sigma} \hat{n}_{i \sigma}, \hat{n}_{i \sigma}=\hat{c}_{i \sigma}^{+} \hat{c}_{i \sigma}, \hat{\rho}_{i}^{+}=\left(\hat{\rho}_{i}^{-}\right)^{\dagger}=\hat{c}_{i \uparrow}^{+} \hat{c}_{i \downarrow}^{+}$ $\hat{c}_{i \sigma}\left(\hat{c}_{i \sigma}^{+}\right)$denotes the annihilation (creation) operator of an electron with spin $\sigma=\uparrow, \downarrow$ at the site $i . \sum_{\langle i, j\rangle}$ indicates the sum over nearest-neighbour sites $i$ and $j$ independently. $z$ denotes the number of nearest neighbours ( $z=2 d$ for $d$-dimensional hypercubic lattice). Finally, $\mu$ is the chemical potential defining concentration of electrons in the system: $n=\frac{1}{N} \sum_{i}\left\langle\hat{n}_{i}\right\rangle$, with $0 \leq n \leq 2$ and $N$ is the total number of lattice sites. $\langle\hat{A}\rangle$ is the average value of operator $\hat{A}$. Model (1) is a generalization of the Penson-Kolb model (e.g. [13-16]) to the case of $U \neq 0$.

It was proven that all interactions between particles on different sites get trivial in the limit of high dimensions $(d \rightarrow+\infty)$ (or equivalently the large coordination number limit: $z \rightarrow+\infty)[17,18]$ and may be treated in the (Hartree) mean-field approximation (MFA):

\footnotetext{
*corresponding author; e-mail: konrad.kapcia@ifpan.edu.pl
}

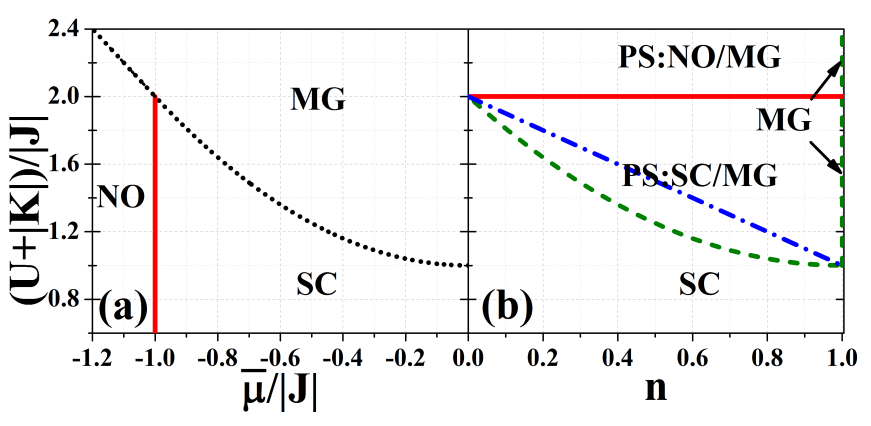

Fig. 1. Ground state diagrams of Hamiltonian $\hat{H}_{A L}$ (atomic limit, $t=0$ ) for $|K| \neq 0$ as a function of $\bar{\mu}=\mu-U / 2$ (a); and as a function of $n$ (b). It is an exact result for $d \rightarrow+\infty$. Details in the text of Sect. 2 .

$$
\hat{\rho}_{i}^{+} \hat{\rho}_{j}^{-} \approx \Delta_{i}^{+} \hat{\rho}_{j}^{-}+\Delta_{j}^{-} \hat{\rho}_{i}^{+}-\Delta_{i}^{+} \Delta_{j}^{-},
$$

where $\Delta_{i}^{+}=\left\langle\hat{\rho}_{i}^{+}\right\rangle$and $\Delta_{j}^{-}=\left\langle\hat{\rho}_{j}^{-}\right\rangle$are the SC order parameters. The Hubbard on-site $U$ interaction is the only interaction which remains dynamical at $d \rightarrow+\infty[17-$ 19]. The relevant question is how accurate the standard broken-symmetry Hartree-Fock mean-field approximation (HFA) (cf. Eq. (3)) for the $U$ term is? The answer to this question is very important because various approximate (in particular mean-field) schemes can introduce different "artificial" effects and could not predict proper solutions [1, 19-21]. For general case of $U \neq 0$ the dynamical mean field theory is an exact one for the standard single-band Hubbard model [19], but it cannot be used for the extended Hubbard model with pair-hopping $J$ term (model (1)).

In this report we check the validity of the HFA for the ground state of model (1) in the limit of very narrow bandwidth $(t \rightarrow 0$ or $U / t \gg 1)$ and $d \rightarrow+\infty$. Section 2 includes the rigorous results obtained in the atomic limit for $d \rightarrow+\infty$. Section 3 is devoted to the discussion of the HFA results for small, but finite $t$. Conclusions 
in Sect. 4 close the presentation. Due to the electronhole symmetry of model (1) the diagrams presented are symmetric under the transformation $n \leftrightarrow 2-n[1,22-24]$.

\section{Results in the atomic limit}

In this section we present a brief review of the zerotemperature results for the atomic limit $(t=0)$ of model (1) derived in [22-25]. For large $U,|J| \gg t$ the effective magnetic interaction in model (1) can be obtained as $K_{\text {eff }} \approx-4 t^{2} /(U-|J|)$. In order to capture the magnetically ordered phases in the atomic limit we additionally introduce effective magnetic interactions $K^{z}$, $K^{x y}$ in the form

$$
\begin{aligned}
& \hat{H}_{M}=-\left(2 K^{z} / z\right) \sum_{\langle i, j\rangle} \hat{s}_{i}^{z} \hat{s}_{j}^{z} \\
& -\left(K^{x y} / z\right) \sum_{\langle i, j\rangle}\left(\hat{s}_{i}^{+} \hat{s}_{j}^{-}+\hat{s}_{j}^{+} \hat{s}_{i}^{-}\right),
\end{aligned}
$$

where $\hat{s}_{i}^{z}=\left(\hat{n}_{i \uparrow}-\hat{n}_{i \downarrow}\right) / 2$, and $\hat{s}_{i}^{+}=\left(\hat{s}_{i}^{-}\right)^{\dagger}=\hat{c}_{i \uparrow}^{+} \hat{c}_{i \downarrow}$ $[21,25]$. In the atomic limit $(t \stackrel{i}{=} 0)$ one can obtain exact results for model $\hat{H}_{A L}=\hat{H}(t=0)+\hat{H}_{M}$ (where $\hat{H}(t=0)$ denotes model (1) with $t=0)$ for $z \rightarrow+\infty$ using variational approach which treats $U$ term exactly and intersite terms within MFA (cf. Eq. (2)) [21-25]. Note that the Coulomb interactions between nearest neighbors have usually higher values than $K$ and $J$ interactions, but only effects of effective $K$ interactions are of our interest in the context of the results for model (1) presented in Sect. 3.

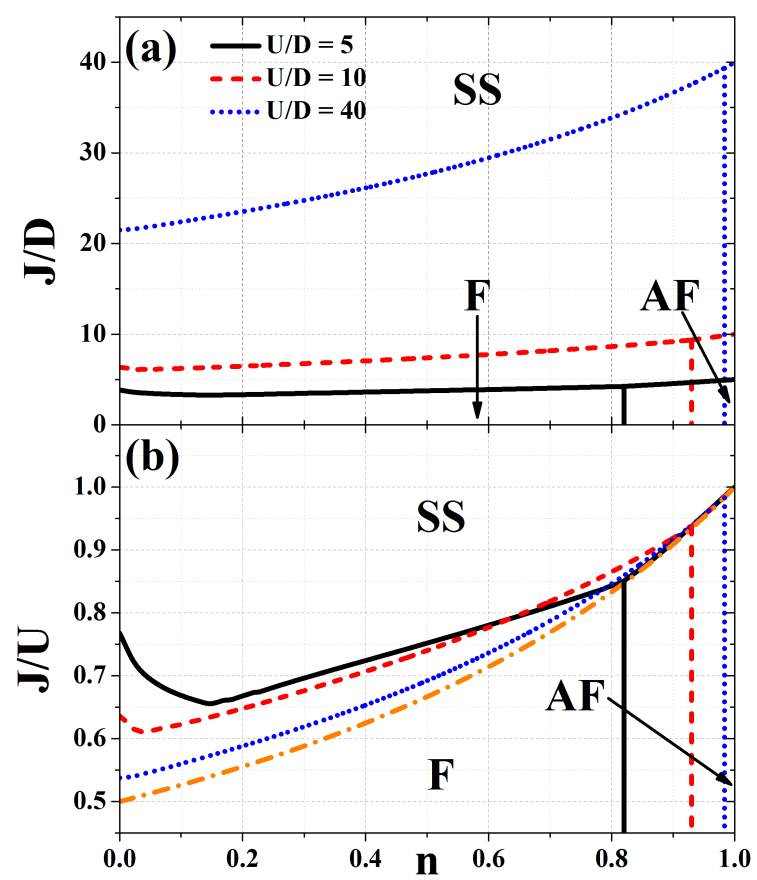

Fig. 2. Ground state diagrams for $D=2 t \neq 0, J>0$, and $U / D=5,10,40$ (solid, dashed and dotted lines, respectively). The dashed-dotted line denotes the rigorous result for the SS-NO boundary in the atomic limit (part (b)).
The ground state phase diagrams for $\hat{H}_{A L}$ are shown in Fig. 1. The model $\hat{H}_{A L}$ exhibits symmetry $J \leftrightarrow$ $-J$ and for $J>0$ the $s$-wave SC (SS) can occur on the diagrams for sufficiently small $(U+|K|) / J(|K|=$ $\left.\max \left\{\left|K^{z}\right|,\left|K^{x y}\right|\right\}\right)$, whereas for $J<0$ the $\eta$-wave SC $(\eta \mathrm{S})$ is stable. For larger $(U+|K|) / J$ the magnetic $(\mathrm{MG})$ phase occurs, which can be either ferromagnetic $(\mathrm{F})$ or antiferromagnetic (AF). If $\left|K^{z}\right|>\left|K^{x y}\right|$ magnetic order occurs in $z$-axis direction ( $\mathrm{F}$ for $K^{z}>0$ and $\mathrm{AF}$ for $\left.K^{z}<0\right)$, if $\left|K^{x y}\right|>\left|K^{z}\right|$ magnetic order is in $x y$-plane $\left(\mathrm{F}\right.$ for $K^{x y}>0$ and $\mathrm{AF}$ for $\left.K^{x y}<0\right)[21,25]$. The SCMG boundary is discontinuous for fixed $\bar{\mu}=\mu-U / 2$ (Fig. 1a) and the phase separated (PS) state occurs: $\mathrm{PS}: \mathrm{SC} / \mathrm{MG}$ in definite range of $n$ (it is coexistence of the SC and MG phases, Fig. 1b). For $|K|=0$ the MG phase changes into the non-ordered (NO) phase and the diagrams preserve their forms shown in Fig. 1 with the change $\mathrm{MG} \rightarrow \mathrm{NO}[22-25]$. The PS:NO/NO state is degenerated with homogeneous NO phase [25].

In Fig. 1b dashed-dotted line denotes the discontinuous boundary between SC and MG phases located at $(U+$ $|K|) /|J|=1+|1-n|$ if the phase separated states are not considered (in such case only homogeneous SC and MG phases can occur on the diagrams, also $c f$. [22-25]). For $|K|=0$ the NO phase occurs.

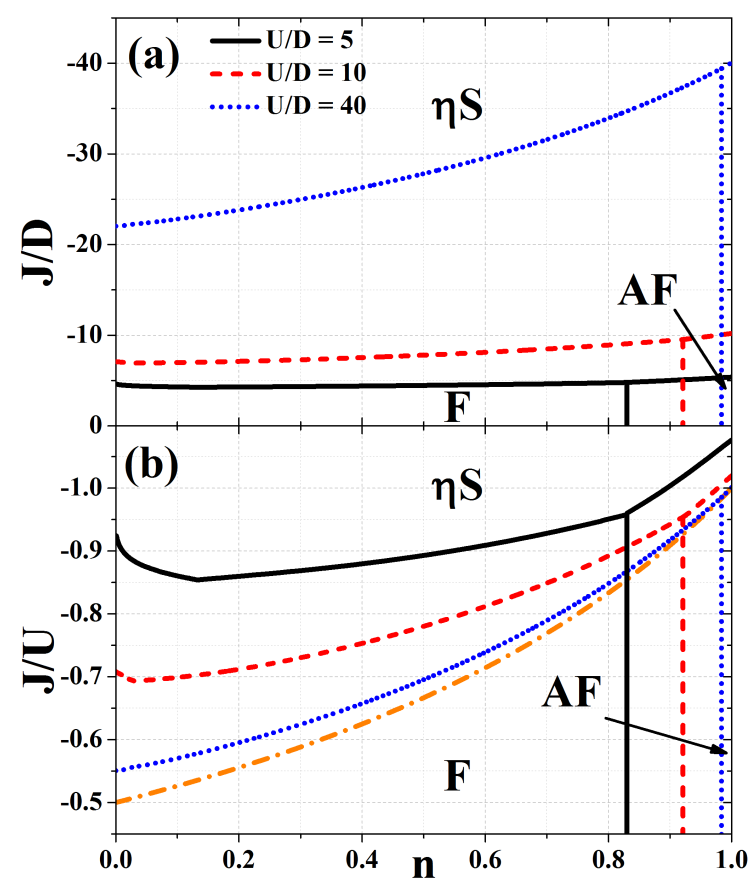

Fig. 3. Ground state diagrams for $D \neq 0, J<0$, and $U / D=5,10,40$ (solid, dashed and dotted lines, respectively). The dashed-dotted line denotes the rigorous result for the $\eta \mathrm{S}-\mathrm{NO}$ boundary in the atomic limit (part (b)).

\section{Diagrams for the finite hopping}

For $t \neq 0$ we use a standard broken-symmetry HartreeFock mean-field approximation for $U$ term in (1) (with 
electron Wick theorem for averages of fermion operators):

$$
\begin{aligned}
& \hat{n}_{i \uparrow} \hat{n}_{i \downarrow}=\hat{c}_{i \uparrow}^{+} \hat{c}_{i \uparrow} \hat{c}_{i \downarrow}^{+} \hat{c}_{i \downarrow} \approx n_{i \uparrow} \hat{n}_{i \downarrow}+n_{i \downarrow} \hat{n}_{i \uparrow}-n_{i \uparrow} n_{i \downarrow} \\
& \quad+\Delta_{i}^{+} \hat{\rho}_{i}^{-}+\Delta_{i}^{-} \hat{\rho}_{i}^{+}-\Delta_{i}^{+} \Delta_{i}^{-}-m_{i}^{+} \hat{s}_{i}^{-}-m_{i}^{-} \hat{s}_{i}^{+} \\
& \quad+m_{i}^{+} m_{i}^{-}
\end{aligned}
$$

where $n_{i \sigma}=\left\langle\hat{n}_{i \sigma}\right\rangle, m_{i}^{+}=\left\langle\hat{s}_{i}^{+}\right\rangle, m_{i}^{-}=\left\langle\hat{s}_{i}^{-}\right\rangle$and $\Delta_{i}^{+}$, $\Delta_{i}^{-}, \hat{s}_{i}^{+}$, as well as $\hat{s}_{i}^{-}$are defined previously. In such an approximation the magnetic orderings in $z$-axis and $x y$ plane are degenerated (i.e. isotropic magnet, the order along any axis in space can occur). For $J$ term the MFA decoupling is applied (Eq. (2)).

In the following the semi-elliptical density of states (SE-DOS) is used: $D(\epsilon)=\frac{2}{\pi D^{2}} \sqrt{1-(\epsilon / D)^{2}}$ for $|\epsilon|<$ $D=2 t$, where $D$ is half-bandwidth. The SE-DOS is an exact result for the Bethe lattice in the limit $z \rightarrow$ $+\infty$ [19]. In general, the advantage of using this DOS is that it is continuous, confined, has sharp band edges and decreases near the edges of band as $\sqrt{\Delta \omega}$, where $\Delta \omega$ is a distance from the band edge. However, the shape of DOS in the limit $t \rightarrow 0$ considered here is not very important.

For better readability and clarity in this section we do not consider phase separated states, which can occur on the phase diagrams ( $c f$. Sect. 2 and [10, 12]). All transitions discussed below are discontinuous ones.

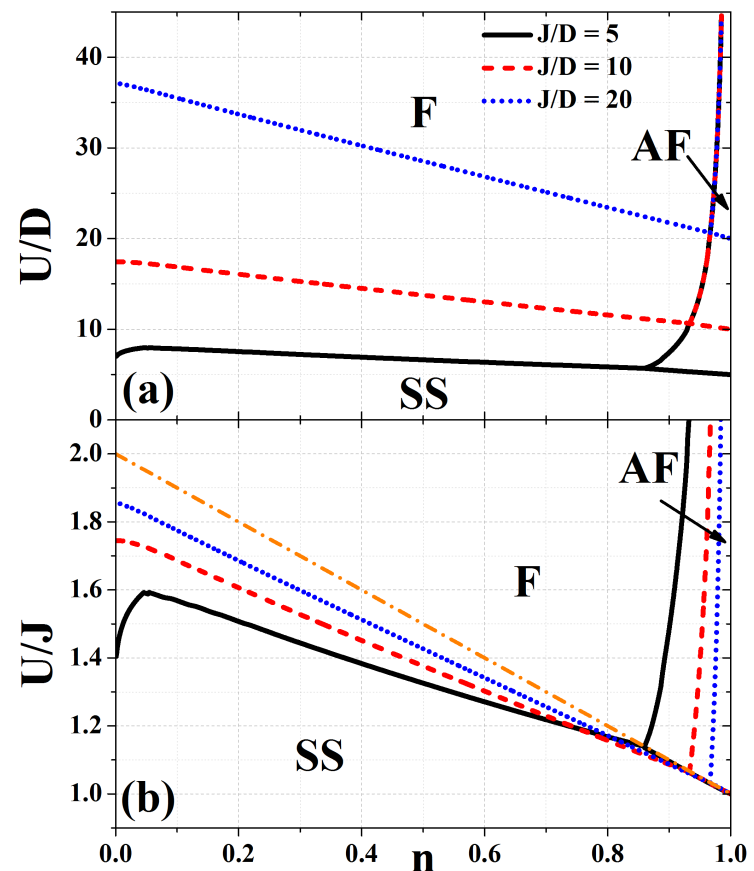

Fig. 4. Ground state diagrams for $D=2 t \neq 0$ and $J / D=5,10,20$ (solid, dashed and dotted lines, respectively). The dashed-dotted line denotes the rigorous result for the SS-NO boundary in the atomic limit (part (b))

For $U \gg D$ (and for $|J| \gg D$ ) the structure of all presented ground state phase diagrams plotted as a function of $n$ are qualitatively similar. On the diagrams three homogeneous phases occur: superconducting (SS for $J>0$

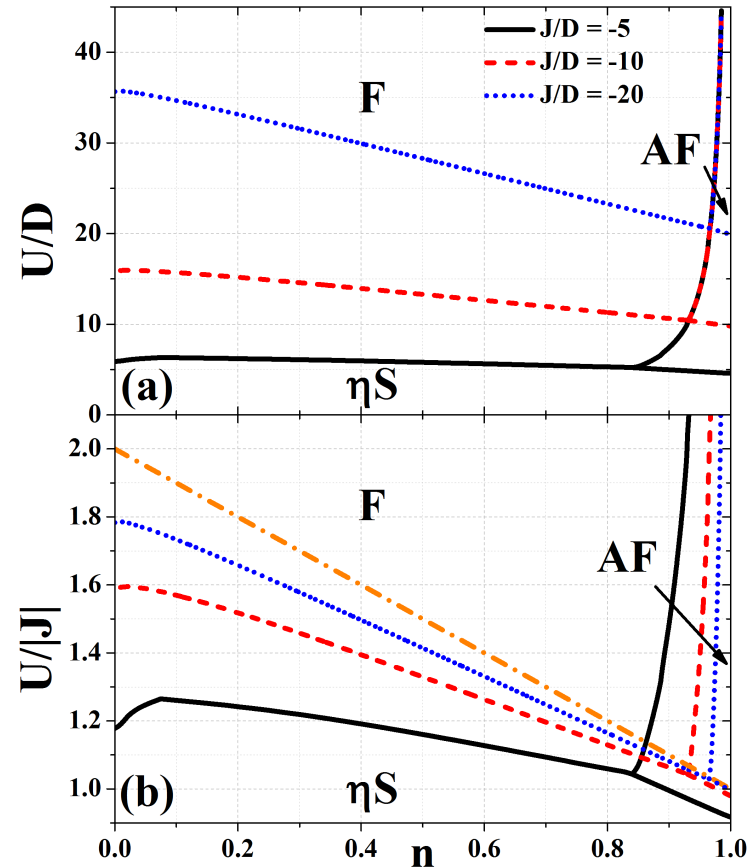

Fig. 5. Ground state diagrams for $D \neq 0$ and $J / D=$ $-5,-10,-20$ (solid, dashed and dotted lines, respectively). The dashed-dotted line denotes the rigorous result for the $\eta \mathrm{S}-\mathrm{NO}$ boundary in the atomic limit (part (b)).

or $\eta \mathrm{S}$ for $J<0$ ), ferromagnetic $(\mathrm{F})$, and antiferromagnetic (AF). The diagrams for fixed values of $U / D \gg 1$ are presented in Fig. 2 (for $J>0$ ) and in Fig. 3 (for $J<0$ ). Figures 4 and 5 are plotted for several fixed positive and negative, respectively, values of $J / D$. The case of large attractive $U(U<0$ and $|U| \gg D)$ is not considered in this paper. It is because negative $U$ stabilizes superconducting phases for any $n$ and only the SC phases occur for $U<0$ (and: $|U| \gg D$ or $|J| \gg D)[1,5,7-12]$.

The magnetic (MG) phases (F or AF) are stabilized by large enough $U /|J|$, whereas large $|J| / U$ favours the $\mathrm{SC}$ phases (SS or $\eta \mathrm{S}$ depending on the sign of $J$ ). The AF phase occurs near $n \simeq 1$ and the $\mathrm{F}$ phase is stable away from half-filling. The SC phases can occur for any $n$. The AF-F boundary depends on $U / D$ (and $n$ ) and is independent of $|J| / D$ for fixed $U / D$. For any $t \neq 0$ the symmetry $J \leftrightarrow-J$ is broken $[6-8,23,24]$, but for $U \gg D$ it is revealed.

It is clearly seen that with increasing $U / D$ the SC-MG boundaries approach to the boundaries derived rigorously at atomic limit $(t=0)(c f$. Figs. $2 \mathrm{~b}$ and $3 \mathrm{~b})$. The boundaries for the SC phases for $t=0$ can be considered as a continuous limit of HFA results in the limit $t \rightarrow 0$ (small but finite $t$ ). However, small $t \neq 0$ introduces magnetic ordering (intersite magnetic interactions $K_{\text {eff }} \approx$ $-4 t^{2} / U$ for $\left.U \gg t,|J|[26]\right)$ and the competition between ferromagnetic and antiferromagnetic order with changing $n$ occurs. The distance between the SC-F boundary (for $t \neq 0$ and fixed: $U / D$ or $J / D$ ) and the SC-NO line (for $t=0$ ) decreases with increasing $n$ (Figs. 2b-5b). Notice 
also that near $n=1$ the SS-AF boundary for $t \neq 0$ is located very near the SS-NO line for $t=0$ (Figs. $2 \mathrm{~b}$ and $4 \mathrm{~b}$ ) in contrary to the $\eta \mathrm{S}-\mathrm{AF}$ line, which is moved towards higher $|J| / U$ (Figs. 3b and $5 \mathrm{~b}$ ).

\section{Concluding remarks}

In this report, we have studied the $t \rightarrow 0$ limit of the Penson-Kolb-Hubbard model at the ground state. For zero-temperature we have evaluated the phase diagrams of the model at fixed $n$ and determined the ranges of the homogeneous phases occurrence in the system for small, but finite, single-electron hopping integral. It is very surprising and interesting that the HFA decoupling of the $U$ term in the limit $t \rightarrow 0$ (but $t \neq 0$ ) gives reasonable results which are in coincidence with exact results for $t=0$ (in the limit $d \rightarrow+\infty$ ) at least in the ground state. Obviously, the HFA for the $U$ term $(U \gg t \neq 0)$ overestimates largely critical temperatures [1].

We have found that the following transitions can occur on the ground state phase diagram of model (1) in the limit $U \gg D(D=2 t \neq 0)$ : (a) $\mathrm{SS} / \eta \mathrm{S} \leftrightarrow \mathrm{F}$, (b) $\mathrm{SS} / \eta \mathrm{S}$ $\leftrightarrow \mathrm{AF}$, and (c) $\mathrm{F} \leftrightarrow \mathrm{AF}$. All these transitions are discontinuous which suggests that the phase separated states have lower energy and they can occur on the diagrams. The study of the diagrams including phase separation for $t \neq 0$ is left for future investigations.

For $U / D \lesssim 2$ or $|J| / D \lesssim 2$ the structure of the diagrams changes significantly $[5-8,10]$ in comparison to the diagrams shown in the present paper, but these ranges of the parameters are beyond the scope of this work.

In this paper we have concentrated only on the location of the phase boundaries. Thus, the difference between free energies of the phases near the boundaries is investigated. We do not discuss the behaviour of the free energy with changing the parameters of the model.

\section{Acknowledgments}

The authors are indebted to Stanisław Robaszkiewicz and Andrzej Ptok for very fruitful discussions during this work and careful reading of the manuscript.

\section{References}

[1] R. Micnas, J. Ranninger, S. Robaszkiewicz, Rev. Mod. Phys. 62, 113 (1990); M. Imada, A. Fujimori, Y. Tokura, Rev. Mod. Phys. 701039 (1998).

[2] D.C. Johnston, Adv. Phys. 59, 803 (2010); P.M. Aswathy, J.B. Anooja, P.M. Sarun, U. Syamaprasad, Supercond. Sci. Technol. 23, 073001 (2010); G.R. Stewart, Rev. Mod. Phys. 83, 1589 (2011).

[3] A. Ptok, J. Phys. Condens. Matter 27, 482001 (2015).

[4] M.M. Wysokiński, M. Abram, J. Spałek, Phys. Rev. B 90, 081114(R) (2014); M. Abram, M.M. Wysokiński, J. Spałek, J. Magn. Magn. Mater. 400, 27 (2016).
[5] A. Hui, S. Doniach, Phys. Rev. B 48, 2063 (1993); A. Belkasri, F.D. Buzatu, Phys. Rev. B 53, 7171 (1996).

[6] G.I. Japaridze, E. Müller-Hartmann, J. Phys. Condens. Matter 9, 10509 (1997); G.I. Japaridze, S. Sarkar, Eur. Phys. J. B 27, 139 (2002).

[7] S. Robaszkiewicz, B.R. Bułka, Phys. Rev. B 59, 6430 (1999); F. Dolcini, A. Montorsi, Phys. Rev. B 62, 2315 (2000).

[8] G.I. Japaridze, A.P. Kampf, M. Sekania, P. Kakashvili, Ph. Brune, Phys. Rev. B 65 , 014518 (2001).

[9] A. Ptok, M.M. Maśka, M. Mierzejewski, J. Phys. Condens. Matter 21, 295601 (2009); A. Ptok, M.M. Maśka, M. Mierzejewski, Phys. Rev. B $\mathbf{8 4}$, 094526 (2011).

[10] W.R. Czart, S. Robaszkiewicz, Acta Phys. Pol. A 127, 275 (2015); W.R. Czart, S. Robaszkiewicz, Acta Phys. Pol. A 127, 278 (2015).

[11] A. Ptok, K.J. Kapcia, Supercond. Sci. Technol. 28, 045022 (2015); A. Ptok, D. Crivelli, K.J. Kapcia, Supercond. Sci. Technol. 28, 045010 (2015).

[12] K.J. Kapcia, W.R. Czart, A. Ptok, J. Phys. Soc. Jpn. 85, 044708 (2016).

[13] K.A. Penson, M. Kolb, Phys. Rev. B 33, 1663 (1986); M. Kolb, K.A. Penson, J. Stat. Phys. 44, 129 (1986).

[14] I. Affleck, J.B. Marston, J. Phys. C 21, 2511 (1988); A.E. Sikkema, I. Affleck, Phys. Rev. B 52, 10207 (1995).

[15] W.R. Czart, S. Robaszkiewicz, Phys. Rev. B 64, 104511 (2001); M. Mierzejewski, M.M. Maśka, Phys. Rev. B 69, 054502 (2004); A. Ptok, M. Mierzejewski, Acta Phys. Pol. A 114, 885 (2008).

[16] S. Robaszkiewicz, W. Czart, Phys. Status Solid $B$ 236, 416 (2003); W.R. Czart, S. Robaszkiewicz, B. Tobijaszewska, Phys. Status Solidi B 244, 2327 (2007).

[17] E. Müller-Hartmann, Z. Phys. B 74, 507 (1989).

[18] P.A. Pearce, C.J. Thompson, Commun. Math. Phys. 41, 191 (1975); P.A. Pearce, C.J. Thompson, Commun. Math. Phys. 58, 131 (1978).

[19] A. Georges, G. Kotliar, W. Krauth, M.J. Rozenberg, Rev. Mod. Phys. 68, 13 (1996).

[20] T. Ayral, S. Biermann, P. Werner, Phys. Rev. B 87, 125149 (2013).

[21] K.J. Kapcia, S. Murawski, W. Kłobus, S. Robaszkiewicz, Physica A 437, 218 (2015).

[22] S. Robaszkiewicz, G. Pawłowski, Physica C 210, 61 (1993).

[23] K. Kapcia, S. Robaszkiewicz, R. Micnas, J. Phys. Condens. Matter 24, 215601 (2012).

[24] K. Kapcia, S. Robaszkiewicz, J. Phys. Condens. Matter 25, 065603 (2013).

[25] K.J. Kapcia, J. Supercond. Nov. Magn. 28, 1289 (2015).

[26] K.A. Chao, J. Spałek, A.M. Oleś, J. Phys. C 10, L271 (1977); K.A. Chao, J. Spałek, A.M. Oleś, Phys. Rev. B 18, 3453 (1978). 